\title{
A Panel of Cancer Testis Antigens and Clinical Risk Factors to Predict Metastasis in Colorectal Cancer
}

\author{
Ramyar Molania, ${ }^{1,2}$ Frouzandeh Mahjoubi, ${ }^{1}$ Rezvan Mirzaei, ${ }^{3}$ \\ Saeed-Reza Khatami, ${ }^{2}$ and Bahar Mahjoubi ${ }^{3}$ \\ ${ }^{1}$ Division of Medical Biotechnology, National Institute of Genetic Engineering and Biotechnology (NIGEB), \\ Pajoohesh Boulevard, Tehran-Karaj Highway, 17th Km, P.O. Box 14965-161, Tehran, Iran \\ ${ }^{2}$ Department of Genetics, Faculty of Science, Shahid Chamran University, Ahwaz, Iran \\ ${ }^{3}$ Hazrat Rasool Hospital, Tehran University of Medical Sciences and Health Care Services, Tehran, Iran \\ Correspondence should be addressed to Frouzandeh Mahjoubi; frouz@nigeb.ac.ir
}

Received 20 August 2013; Revised 30 January 2014; Accepted 31 January 2014; Published 9 March 2014

Academic Editor: Charles Frederick Streckfus

Copyright (C) 2014 Ramyar Molania et al. This is an open access article distributed under the Creative Commons Attribution License, which permits unrestricted use, distribution, and reproduction in any medium, provided the original work is properly cited.

Colorectal cancer (CRC) is the third common carcinoma with a high rate of mortality worldwide and several studies have investigated some molecular and clinicopathological markers for diagnosis and prognosis of its malignant phenotypes. The aim of this study is to evaluate expression frequency of PAGE4, SCP-1, and SPANXA/D cancer testis antigen (CTA) genes as well as some clinical risk markers to predict liver metastasis of colorectal cancer patients. The expression frequency of PAGE4, SCP-1, and SPANXA/D cancer/testis antigen (CTA) genes was obtained using reverse transcription polymerase chain reaction (RT-PCR) assay in 90 colorectal tumor samples including both negative and positive liver metastasis tumors. Statistical analysis was performed to assess the association of three studied genes and clinical risk factors with CRC liver metastasis. The frequency of PAGE4 and $S C P-1$ genes expression was significantly higher in the primary tumours with liver metastasis when statistically compared with primary tumors with no liver metastasis $(P<0.05)$. Among all clinical risk factors studied, the lymph node metastasis and the depth of invasion were statistically correlated with liver metastasis of CRC patients. In addition, using multiple logistic regression, we constructed a model based on PAGE4 and lymph node metastasis to predict liver metastasis of CRC.

\section{Introduction}

Colorectal cancer (CRC) is the third common cancer and the fourth cause of mortality throughout the world [1]. Accumulating evidence shows that CRC can metastasise to many organs and CRC patients die mainly from metastatic disease. Liver is the preferential target of the CRC metastasis [2]. Nearly $10-25 \%$ of CRC patients on diagnosis have liver metastases. However, $20-50 \%$ of CRC patients with no detectable metastasis at the time of resection of the primary tumor will develop liver metastases later on [3-5]. Liver resection in CRC patients with liver metastasis remains the best treatment option and it is associated with a survival rate and a $20-25 \%$ of long-term survivors [6]. However, without treatment, the median overall survival is approximately 9 months after the recognition of liver metastases [6]. To boost the survival rate of CRC patients, selection of patients at high risk for liver metastasis is crucial.

Two of the most important conventional pathological risk factors for diagnosis of colorectal liver metastasis (CLM) are lymph node metastasis and lymphatic invasion [7]. Molecular studies have shown that there are some useful markers for predicting CLM. Several tumor-associated antigens (TAAs) have been identified in colorectal cancer [8-10]. These TTAs may also be presented in normal cells that show lack of tumor specificity. Another category including tumor-specific antigens (TASs) is unique to the tumor and is not produced by any type of normal cells. The most important subgroup of TSA is cancer testis antigens (CTAs) expressed in the normal testis tissue and some types of cancers. Due to their restricted expression in normal adult tissues, CTAs have been attractive targets for biomarker development [11] and 
could serve as unique biomarkers for cancer diagnosis and prognosis [12-15]. CTAs are widely studied in tumors of different histotypes and although some studies have shown that CTA genes are not universally expressed in all tumour types [16-18], based on RT-PCR analysis it has been indicated that various members of CTAs are expressed in different types of cancers such as melanoma, lymphoma, bladder, breast, prostate, kidney, colon, and nonsmall cell lung cancers [1922].

A variety of studies have reported the expression of different members of CTA genes in CRC $[9,23-25]$. The actual frequency of CTA genes expression varies substantially between different studies performed on CRC and we cannot ignore the lack of uniformity in analytical techniques as a source of data variation. Recently, some investigations have been carried out using RT-PCR and statistical analysis to reveal the correlation of some CTA genes expression frequency and clinical risk factors with malignancy in CRCs $[8,26]$. In the current study, implementing the same molecular technique and statistical analysis on our population, we aimed to confirm the impact of three CTA genes expression frequency and some clinical risk factors in both primary tumors with CLM and primary tumors with no CLM in patients with CRC. The selection of these three CTA genes was based on two key reasons: (1) their presence in colorectal cancer tissue or cell lines and (2) their association with tumour aggressiveness $[8,26]$.

\section{Materials and Methods}

2.1. Patients. Ninety patients with locally advanced colorectal cancer admitted to Rasol Akram Hospital in Tehran were enrolled in this study. The project was approved by the local ethics committee of Rasol Akram Hospital and written informed consent was obtained from each case. The colorectal cancer patients had received surgical but not any chemotherapy treatment. During the follow-up period between 2008 and 2013, liver metastases were observed in $36(47 \%)$ cases. Clinicopathological characteristics contained demographic variables (age, gender), tumor size, tumor location (colon and rectum), and pathological status classified according to the TNM system [15]. Fresh tissue specimens including primary tumors with no CLM, primary tumors with CLM tissue, and paired adjacent normal tissue were collected by the clinicians in separated sterile tubes. Tissue samples were frozen and stored at $-70^{\circ} \mathrm{C}$.

2.2. RNA Extraction and cDNA Synthesis. Total RNA extraction was performed from $50-100 \mathrm{mg}$ of each sample with the TriPure Isolation Reagent (Roche Applied Sciences, Germany). For cDNA synthesis, $3 \mu \mathrm{g}$ of total RNA from each sample was used to synthesize first-strand cDNA according to the manufacturer protocol (Fermentas, Germany).

2.3. Reverse Transcriptase-Polymerase Chain Reaction. To evaluate the expression of individual CTA genes, all reactions were carried out in a peqSTAR 96 Universal Thermal Cycler. The PCR mixture included $1 \mu \mathrm{M}$ primer, $200 \mu \mathrm{M}$ of each dNTP (KBC), reaction buffer $1 x$ with $1.5 \mathrm{mM} \mathrm{mgCl}_{2}$, and
1 unit Taq polymerase $(5 \mathrm{U} / \mu \mathrm{L}, \mathrm{KBC})$. The PCR analysis was performed on selected genes using the primers and the conditions shown in Table 1. Amplified segments were analyzed by electrophoresis on a $2.5 \%$ agarose gel, stained with ethidium bromide, and observed under E box vx2 ultraviolet light system.

2.4. Statistical Analysis. All the data were tabulated, and the statistical analysis was performed using the SPSS software V16.0 (SPSS, Inc., Chicago, IL). Association of the frequency of CTA genes expression and clinicopathological markers with CLM was analyzed using Fisher's exact test for a twoby-two contingency table or by the Pearson $\chi^{2}$ test. The multiple logistic regression was performed to determine those independent factors which were significantly important in predicting CLM.

In all statistical analyses, a $P$ value less than 0.05 was considered to be statistically significant. Finally, the coexpression of the CTA genes as well as the association of CTA genes coexpression with CLM was analyzed using Fisher's exact test. All of the variables assessed in this study were categorical except for "age" and "tumour size" and hence to create the two-by-two contingency table, we divided each of them into two groups according to their calculated means (Table 2).

\section{Results}

3.1. Clinical Samples. Samples used in this study including primary tumors without CLM, primary tumors with CLM, and adjacent normal tissues were collected from $90 \mathrm{CRC}$ patients. However, clinicopathological data was available only for 77 patients including 37 (48\%) men and 40 (52\%) women, ranging in age from 17 to 80 years (median 51 years). Table 2 represents the information related to patients' clinicopathological markers.

3.2. Results of RT-PCR Amplification. CTA mRNA expression was examined with RT-PCR in primary tumors, liver metastasis, and paired adjacent normal tissues in each CRC patient. None of these genes were expressed in normal mucosal tissues. Figure 1 illustrates positive RT-PCR results for each CTA gene studied.

3.3. Correlation between CTA mRNA Expression and CLM. The results of Pearson $\chi^{2}$ test and fisher exact test showed higher frequency of PAGE4 and SCP-1 genes expression in primary tumors with CLM than that in primary tumors without CLM and the difference was statistically significant $(P<0.05)$. SPANXA/D gene expression was also detected at higher frequencies in primary tumors with CLM, but the difference with primary tumors without CLM was not statistically significant. Nevertheless, there was no significant difference in expression patterns of CTA genes between these two categories of CRC (Table 3).

3.4. Correlation between CTA mRNA Expression and Clinicopathological Markers. The association between clinical risk factors and the expression frequency of three CTA genes were 
TABLE 1: Primers and condition of RT-PCR analysis.

\begin{tabular}{|c|c|c|c|c|c|}
\hline \multirow{2}{*}{ Genes } & \multirow{2}{*}{ Primers sequence $\left(5^{\prime} \rightarrow 3^{\prime}\right)$} & \multicolumn{4}{|c|}{ Conditions } \\
\hline & & Denaturation & Annealing & Extension & Cycle (no.) \\
\hline PAGE4 & $\begin{array}{l}\text { GATGTGGTTGTATTCGTG } \\
\text { ATCTCCTGCTTCTTTAGTC }\end{array}$ & $94^{\circ} \mathrm{C}-1 \mathrm{~min}$ & $57^{\circ} \mathrm{C}-30 \mathrm{~s}$ & $72^{\circ} \mathrm{C}-1 \mathrm{~min}$ & 35 \\
\hline$S C P-1$ & $\begin{array}{l}\text { CCAAAGGCATATACAGTGAAGA } \\
\text { CAGGGGTTGTTAGAGATGAAGG }\end{array}$ & $94^{\circ} \mathrm{C}-1 \mathrm{~min}$ & $62^{\circ} \mathrm{C}-45 \mathrm{~s}$ & $72^{\circ} \mathrm{C}-1 \mathrm{~min}$ & 35 \\
\hline SPANXA/D & $\begin{array}{l}\text { GACAAACAATCCAGTGCC } \\
\text { TCCTCCTGTAGCGAACCA }\end{array}$ & $94^{\circ} \mathrm{C}-1 \mathrm{~min}$ & $57^{\circ} \mathrm{C}-1 \mathrm{~min}$ & $72^{\circ} \mathrm{C}-1 \mathrm{~min}$ & 35 \\
\hline
\end{tabular}

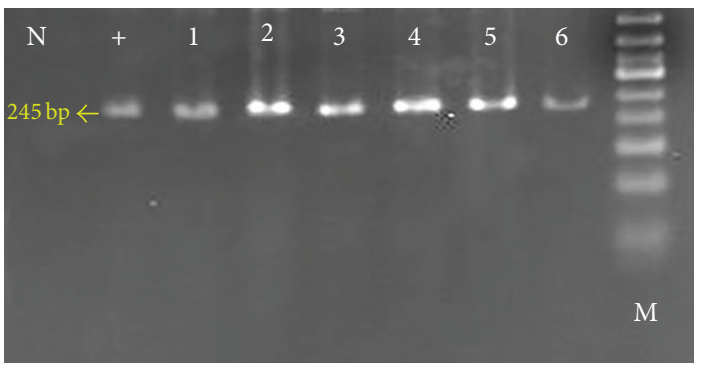

(a)

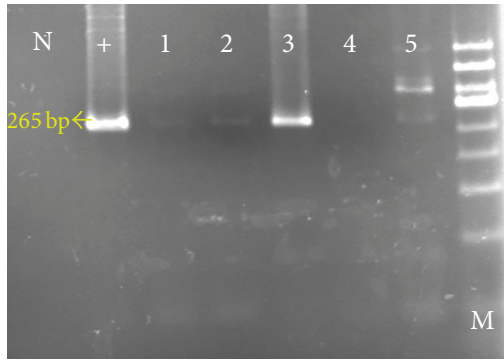

(b)

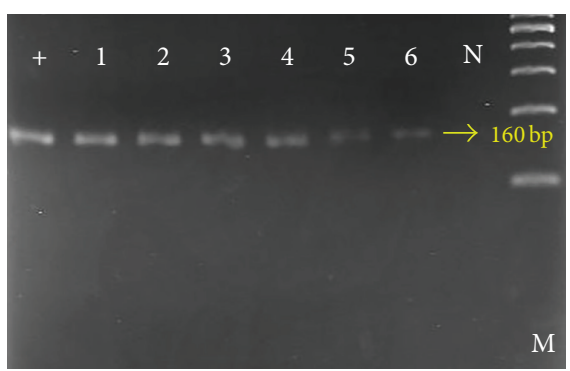

(c)

FIGURE 1: The figures show the result of RT-PCR analysis of positive mRNA expression of CTA genes in some patients. Each band represents a positive mRNA expression of CTA genes: (a), (b), and (c): PAGE4, SCP-1, and SPANXA/D, respectively. M: molecular marker; N: negative control; +: positive control.

examined (Table 3). Our results revealed that the expression of SCP-1 and PAGE4 was significantly correlated with lymph node metastasis ( $\mathrm{N}$ category) $(P<0.05)$.

3.5. Clinicopathological Parameters in Patients with CLM. We evaluated the correlation between clinical risk factors and primary tumours with CLM. The statistical results suggested that the depth of invasion $(P=0.011)$ and lymph node metastasis $(P=0.001)$ were associated with liver metastasis of CRC.

3.6. Coexpression of CTA Genes in CRC. Of all 90 CRC patients, 46 patients (51\%) expressed at least one CT gene. Among them, 23 patients (50\%) expressed one CT gene and the other 23 patients (50\%) expressed two or three CT genes. Only the expression of $S C P-1$ was significantly associated with PAGE4 and SPANXA/D genes expression $(P<0.05)$ (Table 4). in Patients with CRC. In this study, $48 \%$ of patients with
CRC showed CLM, among which $17 \%$ had no expression of three genes, $28 \%$ had coexpression of two genes, and $11 \%$ had coexpression of three genes (Table 5).

3.8. Binary Logistic Regression Analysis. Binary logistic regression analysis with maximum likelihood estimation was accomplished with 4 potential risk factors for liver metastasis (SCP-1, PAGE4, depth of invasion, and lymph node involvement). We found a statistically significant relationship between both PAGE4 $\left(X_{1}\right)$ and lymph node involvement (N1: $\left.X_{2}, \mathrm{~N} 2: X_{3}\right)$ with CLM $(P<0.05)$ (Table 6) and therefore they were included in the multiple logistic regression model for liver metastasis risk prediction. PAGE4 and lymph node involvement were identified as independent risk factors for CLM $(P<0.05)$.

The following equation was used to predict estimated risk of liver metastasis in CRC patients:

$$
P x=\frac{e^{\left(-2.497+2.627 X_{1}+2.373 X_{2}+2.249 X_{3}\right)}}{1+e^{\left(-2.497+2.627 X_{1}+2.373 X_{2}+2.249 X_{3}\right)}} .
$$


The classification accuracy rate of the model based on PAGE4 and lymph node metastases was $80.3 \%$ which surpassed the proportional by chance accuracy criteria, supporting the utility of the model. Table 7 shows predicted risk of liver metastases based on possible results of the model compared with actual observation of liver metastasis from CRC patients in this dataset.

In patients with none of the two PAGE4 and lymph node metastases markers, the actual percentage of CRC patients with liver metastasis was $6.9 \%$, whereas the predicted risk based on the model was 7.6\%. When lymph node involvement, depending on the type of $\mathrm{N}$ category $(N 1, N 2)$, was present, the actual percentage of CRC patients with liver metastasis ranged from $43 \%$ to $50 \%$, and predicted risk ranged from $44 \%$ to $46 \%$. When both risk factors existed, the actual proportion of CLM was $94 \%$, and risk calculated by the model was $92 \%$. Thus, adding PAGE4 to the classical risk factor of lymph node involvement improved the predictive power for liver metastasis by nearly $40 \%$.

\section{Discussion}

Colorectal cancer is the third common carcinoma with a high rate of mortality around the world, corresponding to the second cause of cancer-related death [27]. In most cases, death results from the formation of secondary neoplasms called metastases. Over the last decades, tremendous studies about cancer molecular markers have been accomplished; however, only a few such markers have entered clinical practice. The lack of clinical prognostic markers clearly reflects limitations in prognostic studies. On the other hand, between-study heterogeneity in evaluating the role of biomarkers in cancer necessitates confirmatory studies to validate primary studies.

To date, about 83 CTA gene families including more than 140 members have been discovered [28]. Although the function of CTA genes is still largely unknown partly due to their presence in multiple tumor types, their limited expression in normal tissue has made them ideal molecular markers for cancer prognosis and diagnosis [29, 30]. To improve the prognosis of CRC, the most significant considerations are the selection of patients at high risk for liver metastasis and subsequently the initiation of suitable adjuvant therapy. Adjuvant therapy in patients with CRC after curative resection has been reported to be useful for improving overall and disease-free survival $[31,32]$.

Although, some scientists initially hypothesized that there must be an obvious difference in gene expression profiles between primary tumours with no CLM and primary tumours with CLM (because they assumed that primary cancer cells must obtain the potential of metastasis during late tumorigenesis steps), in this study we found no apparent difference in CTA expression pattern between these two categories of CRC. This finding confirmed that CTA genes expression pattern is maintained during CRC liver metastasis. Our results are consistent with the studies of Chen et al. and Alves et al., who reported no difference in expression pattern of understudied CTA genes between primary tumours with no CLM and primary tumours with
TAble 2: Patients' clinicopathological data.

\begin{tabular}{|c|c|c|}
\hline \multirow[b]{2}{*}{ Risks factors } & \multicolumn{2}{|c|}{ Tumour tissues } \\
\hline & $\begin{array}{c}\text { Negative liver } \\
\text { metastases } 53(41)\end{array}$ & $\begin{array}{c}\text { Positive liver } \\
\text { metastases } 47 \text { (36) }\end{array}$ \\
\hline \multicolumn{3}{|l|}{ Gender } \\
\hline Male $(n=37)$ & $53(22)$ & $41(15)$ \\
\hline Female $(n=40)$ & 47 (19) & $59(21)$ \\
\hline \multicolumn{3}{|l|}{ Age } \\
\hline$>51(n=41)$ & $53(22)$ & $52(19)$ \\
\hline$<51(n=36)$ & 47 (19) & $48(17)$ \\
\hline \multicolumn{3}{|l|}{ Tumor size } \\
\hline$<5(n=40)$ & $44(18)$ & $61(22)$ \\
\hline$\geq 5(n=36)$ & $66(22)$ & $39(14)$ \\
\hline \multicolumn{3}{|l|}{ Tumor location } \\
\hline Colon (32) & $39(16)$ & $45(16)$ \\
\hline Rectum (45) & $61(25)$ & $55(20)$ \\
\hline \multicolumn{3}{|l|}{$\begin{array}{l}\text { Depth of tumor } \\
\text { invasion* ( } \mathrm{T} \text { factor) }\end{array}$} \\
\hline $\mathrm{T} 1(n=0)$ & 0 & 0 \\
\hline $\mathrm{T} 2(n=25)$ & $49(20)$ & $14(5)$ \\
\hline T3 $(n=45)$ & $46(19)$ & $72(26)$ \\
\hline $\mathrm{T} 4(n=7)$ & $5(2)$ & $14(5)$ \\
\hline \multicolumn{3}{|c|}{$\begin{array}{l}\text { Lymph node } \\
\text { metastasis }^{*} \text { ( } \mathrm{N} \text { factor) }\end{array}$} \\
\hline N0 $(n=38)$ & $76(31)$ & $19(7)$ \\
\hline $\mathrm{N} 1(n=25)$ & $12(5)$ & $56(20)$ \\
\hline $\mathrm{N} 2(n=14)$ & $12(5)$ & $25(9)$ \\
\hline
\end{tabular}

Data are percentage of patients with number in parentheses.

${ }^{*} P<0.05$ for the comparison primary tumors without CLM versus primary tumors with CLM.

CLM [8, 9]. Moreover, these two studies have shown that there are similar expression profiles in the two CRC categories obtained from the same individuals. Also, another study on breast cancer has delineated that metastasis development in individuals is related to their different genetic backgrounds; in other words, metastasis may be unavoidable for patients with certain genetic profiles, while for others, it may never arise [33]. Our results together with these studies have thrown the initial hypothesis into question.

According to our findings, there is a statistically significant higher frequency in expression of PAGE4 and SCP-1 genes in primary tumours with CLM when comparing to primary tumors with no CLM $(P<0.05)$, suggesting that the expression of these two genes might have a correlation with the process of liver metastasis. This result confirms the study outcome of Chen et al. who reported that the expression frequency of PAGE4 and SCP-1 genes in colorectal cancer has significant correlation with liver metastasis [8]. Some studies on other types of cancer also revealed similar results for the association of PAGE4 gene expression with aggressive phenotypes $[34,35]$. In addition, a recent study has shown that silencing and overexpressing of PAG4 mRNA in prostate cancer xenografts are linked, respectively, to 
TABLE 3: Correlation between clinicopathologic risk factors and expression frequency of CTA.

\begin{tabular}{|c|c|c|c|c|c|c|c|c|c|}
\hline \multirow{2}{*}{ Risk factors } & \multicolumn{3}{|c|}{ PAGE4 } & \multicolumn{3}{|c|}{$S C P-1$} & \multicolumn{3}{|c|}{ SPANXA1 } \\
\hline & - & + & $P$ value & - & + & $P$ value & - & + & $P$ value \\
\hline \multicolumn{10}{|l|}{ Gender } \\
\hline Male $(n=37)$ & 59 & 41 & \multirow{2}{*}{0.818} & 76 & 24 & \multirow{2}{*}{0.617} & 16 & 84 & \multirow{2}{*}{1} \\
\hline Female $(n=40)$ & 55 & 45 & & 70 & 30 & & 15 & 85 & \\
\hline \multicolumn{10}{|l|}{ Age } \\
\hline$>51(n=41)$ & 54 & 46 & \multirow{2}{*}{0.645} & 71 & 29 & \multirow{2}{*}{0.799} & 80 & 20 & \multirow{2}{*}{0.645} \\
\hline$<51(n=36)$ & 61 & 39 & & 75 & 25 & & 89 & 11 & \\
\hline \multicolumn{10}{|l|}{ Tumor location } \\
\hline Colon (32) & 40 & 60 & \multirow{2}{*}{0.493} & 49 & 51 & \multirow{2}{*}{0.262} & 30 & 70 & \multirow{2}{*}{0.365} \\
\hline Rectum (45) & 37 & 63 & & 60 & 40 & & 38 & 62 & \\
\hline \multicolumn{10}{|l|}{$\mathrm{T}$ category } \\
\hline $\mathrm{T} 1(n=0)$ & 0 & 0 & \multirow{4}{*}{0.057} & 0 & 0 & \multirow{4}{*}{0.143} & 0 & 0 & \multirow{4}{*}{0.482} \\
\hline $\mathrm{T} 2(n=24)$ & 75 & 25 & & 79 & 21 & & 83 & 17 & \\
\hline $\mathrm{T} 3(n=45)$ & 53 & 47 & & 76 & 24 & & 82 & 18 & \\
\hline $\mathrm{T} 4(n=7)$ & 29 & 71 & & 43 & 57 & & 100 & 0 & \\
\hline \multicolumn{10}{|l|}{ M category } \\
\hline M0 $(n=41)$ & 85 & 15 & \multirow{2}{*}{$0.001^{*}$} & 85 & 15 & \multirow{2}{*}{$0.011^{*}$} & 85 & 15 & \multirow{2}{*}{1} \\
\hline $\mathrm{M} 1(n=36)$ & 25 & 75 & & 62 & 38 & & 83 & 17 & \\
\hline \multicolumn{10}{|l|}{$\mathrm{N}$ category } \\
\hline N0 $(n=38)$ & 76 & 24 & \multirow{3}{*}{$0.002^{*}$} & 87 & 13 & \multirow{3}{*}{$0.003^{*}$} & 92 & 8 & \multirow{3}{*}{0.181} \\
\hline $\mathrm{N} 1(n=25)$ & 28 & 72 & & 48 & 52 & & 76 & 24 & \\
\hline $\mathrm{N} 2(n=14)$ & 57 & 43 & & 79 & 21 & & 79 & 21 & \\
\hline \multicolumn{10}{|l|}{ Tumor size } \\
\hline$<5(n=38)$ & 53 & 47 & \multirow{2}{*}{0.355} & 74 & 26 & \multirow{2}{*}{0.798} & 80 & 20 & 0623 \\
\hline$\geq 5(n=36)$ & 64 & 36 & & 69 & 31 & & 67 & 33 & \\
\hline
\end{tabular}

Data are percentages of patients with (+) or without (-) the gene expression.

${ }^{*}$ Statistically significant, $P<0.05$.

TABLE 4: Association between the expressions of three CTA genes.

\begin{tabular}{|c|c|c|c|c|c|c|}
\hline \multirow{2}{*}{ CTA genes } & \multicolumn{2}{|c|}{$S C P-1$} & \multirow{2}{*}{$P$ value } & \multicolumn{2}{|c|}{ SPANAXA/D } & \multirow{2}{*}{$P$ value } \\
\hline & + & - & & + & - & \\
\hline \multicolumn{7}{|l|}{ PAGE4 } \\
\hline+ & 45 & 55 & \multirow{2}{*}{$0.004^{*}$} & 21 & 79 & \multirow{2}{*}{0.342} \\
\hline- & 14 & 86 & & 11 & 89 & \\
\hline \multicolumn{7}{|l|}{$S C P-1$} \\
\hline+ & & & & 48 & 52 & \multirow{2}{*}{$0.000^{*}$} \\
\hline- & & & & 4 & 96 & \\
\hline
\end{tabular}

Data are percentages of patients with $(+)$ or without $(-)$ the gene expression. ${ }^{*}$ Statistically significant, $P<0.05$.

induction of cell death via apoptosis and protection of cells from stress-induced death [36]. The correlation between higher frequency of SCP-1 gene expression and high graded tumor was also reported by Tammela et al. who worked on epithelial ovarian cancer (EOC) [37].

According to our statistical analysis, there was no significant difference of $S P A N X A / D$ gene expression frequency between primary tumours with CLM and primary tumours with no CLM. In contrast, Chen et al. reported that the frequency of SPANX gene expression was significantly higher in primary tumours with CLM than that in primary tumours with no CLM though they did not determine the subfamily of SPANX that they studied [8].

The association between CTA genes expression frequency and clinicopathological factors was also examined and our data showed that the expression of PAGE4 and SCP-1 was correlated with the presence of lymph node metastasis. Kong et al. in a study on PAGE4 demonstrated that the expression frequency of this gene was correlated with lymph node metastasis in gastric cancer patients [34], while in a study on colorectal cancer, it has been reported that there was no significant correlation between PAGE4 gene expression frequency and lymph node metastases [8]. In terms of SCP1 gene, Chen et al. demonstrated that there is a significant correlation between its expression frequency and the presence of lymph node metastasis in patients with CRC [8]. Therefore, our findings suggested that both PAGE4 and SCP1 genes expression frequencies are associated with malignant phenotypes in CRC, especially during both local invasion and distant metastasis. However, the expression frequency of all three CTA genes was independent of other clinical factors (Table 1).

Of all clinicopathological factors investigated in this study, the frequency of lymph node metastasis and depth of tumour invasion were significantly correlated with CLM. This 
TABLE 5: Association of coexpression of 3 cancer/testis antigens with liver metastasis.

\begin{tabular}{|c|c|c|c|c|c|}
\hline \multirow{2}{*}{ Tumour tissues } & \multicolumn{5}{|c|}{ Expression statute } \\
\hline & No expression & One CTA expression & Two CTA expressions & Three CTA expressions & $P$ value \\
\hline Negative liver metastases CRC (M0) & 74 & 17 & 2 & 7 & $0 *$ \\
\hline Positive liver metastases CRC (M1) & 17 & 44 & 28 & 11 & \\
\hline
\end{tabular}

Data are percentages of patients.

${ }^{*}$ Statistically significant, $P<0.05$.

TABLE 6: Binary logistic regression analysis of expression of PAGE4 $\left(X_{1}\right)$ and lymph node involvement (N1, N2).

\begin{tabular}{|c|c|c|c|c|c|c|}
\hline & Regression coefficient & SE & Odd ratio & \multicolumn{2}{|c|}{ 95\% Confidence interval } & $P$ value \\
\hline PAGE4 $\left(X_{1}\right)$ & 2.627 & 0.682 & 13.833 & 3.634 & 52.650 & $0.000^{*}$ \\
\hline $\mathrm{N} 1\left(X_{2}\right)$ & 2.373 & 0.762 & 10.735 & 2.409 & 47.842 & $0.002^{*}$ \\
\hline $\mathrm{N} 2\left(X_{3}\right)$ & 2.249 & 0.834 & 9.483 & 1.849 & 48.635 & $0.007^{*}$ \\
\hline Constant & -2.497 & 0.601 & 0.082 & & & \\
\hline
\end{tabular}

SE: standard error.

${ }^{*}$ Statistically significant, $P<0.05$.

TABLE 7: Possible combinations of PAGE4 and lymph node involvement (N1, N2) for assessing the utility of the model.

\begin{tabular}{|c|c|c|c|c|c|c|}
\hline Possible combination & PAGE4 & $\mathrm{N} 2$ & N1 & Number of patients & Actual risk \% & Predicted risk \% \\
\hline 1 & - & - & - & 29 & 6.9 & 7.6 \\
\hline 2 & - & - & + & 7 & 43 & 46 \\
\hline 3 & - & + & - & 8 & 50 & 44 \\
\hline 4 & + & - & - & 9 & 56 & 53 \\
\hline 5 & + & + & - & 6 & 83 & 92 \\
\hline 6 & + & - & + & 17 & 94 & 92 \\
\hline
\end{tabular}

finding supports the result obtained from the study of Chen et al. [8].

Interestingly, some reports suggested that multiple CTA genes tend to be coexpressed in the same tumor. $\mathrm{Li}$ et al. by investigating coexpression of some CTA genes in 121 CRC patients revealed that $56.2 \%$ expressed at least one CT gene. Among them, 28.9\% expressed one CT gene and $27.3 \%$ expressed more than two CT genes [14]. Our study on 90 CRC patients showed that $51 \%$ expressed at least one CT gene. Among them, 50\% expressed one CT gene and 50\% expressed two or three CT genes. We also assessed the correlation of these coexpressions between primary tumours with CLM and primary tumours with no CLM. The results showed that coexpression of CTA genes was higher in primary tumours with CLM than in primary tumours with no CLM and the difference was statistically significant $(P<0.001)$.

In the current confirmatory study, we established a formula to predict liver metastasis in patients with CRC. All factors significantly correlated with liver metastasis (the frequency of PAGE4 and SCP-1 genes expression, depth of invasion, and lymph node metastasis) were taken into account for performing multiple logistic regression analysis. However, only two independent factors-PAGE4 and lymph node metastasis-were selected as candidate markers for establishing a panel to predict liver metastasis in CRC patients. The classification accuracy of the model was $80.3 \%$ and this panel could show high similarity between observed and predicted risk of liver metastasis (Table 7). Interestingly, adding PAGE4 to the clinical risk factor of lymph node involvement could improve the power of liver metastasis prediction by approximately $40 \%$. The regression model constructed by Chen et al. was based on three independent risk factors including PAGE4, lymph node metastasis, and vessel cancer embolus status. According to their report, involving PAGE4to the two classical risk factors (vessel cancer embolus and lymph node metastasis) improved the predictive power for liver metastasis only by nearly $20 \%$ [8].

In conclusion, we could confirm the significant association between the frequency of PAGE4 and SCP-1 genes expression and CRC liver metastasis; however, we did not verify this association for SPANXA/D. We also confirmed that the frequency of lymph node metastasis and depth of tumour invasion were significantly correlated with CRC liver metastasis. Although we verified the correlation between SCP-1 and lymph node metastasis, the lack of correlation between PAGE4 and lymph node metastasis was rejected in our dataset.

We believe that constructing a more accurate predictive model based on molecular markers and their subsequent validation can bring a new horizon in diagnosis/prognosis of colorectal cancer and the management of cancer patients.

\section{Conflict of Interests}

The authors declare that there is no conflict of interests regarding the publication of this paper. 


\section{References}

[1] F. A. Haggar and R. P. Boushey, "Colorectal cancer epidemiology: incidence, mortality, survival, and risk factors," Clinics in Colon and Rectal Surgery, vol. 22, no. 4, pp. 191-197, 2009.

[2] N. C. Bird, D. Mangnall, and A. W. Majeed, "Biology of colorectal liver metastases: a review," Journal of Surgical Oncology, vol. 94, no. 1, pp. 68-80, 2006.

[3] W. J. H. J. Meijerink, M. H. G. M. Van Der Pas, D. L. Van Der Peet, M. A. Cuesta, and S. Meijer, "New horizons in colorectal cancer surgery," Surgical Endoscopy and Other Interventional Techniques, vol. 23, no. 1, pp. 1-3, 2009.

[4] B. Nordlinger, M.-A. Quilichini, and R. Parc, "Hepatic resection for colorectal liver metastases: influence on survival of preoperative factors and surgery for recurrences in 80 patients," Annals of Surgery, vol. 205, no. 3, pp. 256-263, 1987.

[5] G. P. Kanas, A. Taylor, J. N. Primrose et al., "Survival after liver resection in metastatic colorectal cancer: review and metaanalysis of prognostic factors," Journal of Clinical Epidemiology, vol. 4, pp. 283-301, 2012.

[6] T. Ruers and R. P. Bleichrodt, "Treatment of liver metastases, an update on the possibilities and results," European Journal of Cancer, vol. 38, no. 7, pp. 1023-1033, 2002.

[7] J. Weitz, P. Kienle, A. Magener et al., "Detection of disseminated colorectal cancer cells in lymph nodes, blood and bone marrow," Clinical Cancer Research, vol. 5, no. 7, pp. 1830-1836, 1999.

[8] Z. Chen, M. Li, Y. Yuan, Q. Wang, L. Yan, and J. Gu, "Cancer/testis antigens and clinical risk factors for liver metastasis of colorectal cancer: a predictive panel," Diseases of the Colon and Rectum, vol. 53, no. 1, pp. 31-38, 2010.

[9] P. M. S. Alves, N. Lévy, H. Bouzourene et al., "Molecular and immunological evaluation of the expression of cancer/testis gene products in human colorectal cancer," Cancer Immunology, Immunotherapy, vol. 56, no. 6, pp. 839-847, 2007.

[10] F. Bettoni, F. C. Filho, D. M. Grosso et al., "Identification of FAM46D as a novel cancer/testis antigen using EST data and serological analysis," Genomics, vol. 94, no. 3, pp. 153-160, 2009.

[11] J. J. Kim, K. Rajagopalan, B. Hussain et al., "CETN1 is a cancer testis antigen with expression in prostate and pancreatic cancers," Biomarker Research, vol. 1, article 22, 2013.

[12] E. Fratta, S. Coral, A. Covre et al., "The biology of cancer testis antigens: putative function, regulation and therapeutic potential," Molecular Oncology, vol. 5, no. 2, pp. 164-182, 2011.

[13] M. Chiriva-Internati, Y. Yu, L. Mirandola et al., "Identification of AKAP-4 as a new cancer/testis antigen for detection and immunotherapy of prostate cancer," Prostate, vol. 72, no. 1, pp. 12-23, 2012.

[14] L. Mirandola, M. J. Cannon, E. Cobos et al., "Cancer testis antigens: novel biomarkers and targetable proteins for ovarian cancer," International Reviews of Immunology, vol. 30, no. 2-3, pp. 127-137, 2011.

[15] M. van Duin, A. Broyl, Y. de Knegt et al., "Cancer testis antigens in newly diagnosed and relapse multiple myeloma: prognostic markers and potential targets for immunotherapy," Haematologica, vol. 96, no. 11, pp. 1662-1669, 2011.

[16] L. G. Almeida, N. J. Sakabe, A. R. de Oliveira et al., "CTdatabase: a knowledge-base of high-throughput and curated data on cancer-testis antigens," Nucleic Acids Research, vol. 37, no. 1, pp. D816-D819, 2009.

[17] Y. H. Cheng, E. W. Wong, and C. Y. Cheng, "Cancer/testis (CT) antigens, carcinogenesis and spermatogenesis," Spermatogenesis, vol. 1, no. 3, pp. 209-220, 2011.
[18] F. F. Costa, K. Le Blanc, and B. Brodin, "Concise review: cancer/testis antigens, stem cells, and cancer," Stem Cells, vol. 25, no. 3, pp. 707-711, 2007.

[19] M. J. Scanlan, A. O. Gure, A. A. Jungbluth, L. J. Old, and Y.-T. Chen, "Cancer/testis antigens: an expanding family of targets for cancer immunotherapy," Immunological Reviews, vol. 188, pp. 22-32, 2002.

[20] M. J. Scanlan, A. J. G. Simpson, and L. J. Old, "The cancer/testis genes: review, standardization, and commentary," Cancer Immunity, vol. 4, article 1, 2004.

[21] L. Calabrò, E. Fonsatti, M. Altomonte et al., "Methylationregulated expression of cancer testis antigens in primary effusion lymphoma: immunotherapeutic implications," Journal of Cellular Physiology, vol. 202, no. 2, pp. 474-477, 2005.

[22] V. Gattei, E. Fonsatti, L. Sigalotti et al., "Epigenetic immunomodulation of hematopoietic malignancies," Seminars in Oncology, vol. 32, no. 5, pp. 503-510, 2005.

[23] J. Choi and H. Chang, "The expression of MAGE and SSX, and correlation of COX2, VEGF, and survivin in colorectal cancer," Anticancer Research, vol. 32, no. 2, pp. 559-564, 2012.

[24] A. G. Dakshinamurthy, R. Ramesar, P. Goldberg, and J. M. Blackburn, "Infrequent and low expression of cancer-testis antigens located on the X chromosome in colorectal cancer: Implications for immunotherapy in South African populations," Biotechnology Journal, vol. 3, no. 11, pp. 1417-1423, 2008.

[25] T. Hanafusa, A. E. A. Mohamed, S. Domae, E. Nakayama, and T. Ono, "Serological identification of Tektin5 as a cancer/testis antigen and its immunogenicity," BMC Cancer, vol. 12, article $520,2012$.

[26] G. Almanzar, P. B. Olkhanud, M. Bodogai et al., "Sperm-derived SPANX-B is a clinically relevant tumor antigen that is expressed in human tumors and readily recognized by human $\mathrm{CD} 4^{+}$and $\mathrm{CD}^{+}$T cells," Clinical Cancer Research, vol. 15, no. 6, pp. 19541963, 2009.

[27] B. M. Wolpin and R. J. Mayer, "Systemic treatment of colorectal cancer," Gastroenterology, vol. 134, no. 5, pp. 1296-1310, 2008.

[28] A. J. G. Simpson, O. L. Caballero, A. Jungbluth, Y.-T. Chen, and L. J. Old, "Cancer/testis antigens, gametogenesis and cancer," Nature Reviews Cancer, vol. 5, no. 8, pp. 615-625, 2005.

[29] K. Odunsi, F. Qian, J. Matsuzaki et al., "Vaccination with an NYESO-1 peptide of HLA class I/II specificities induces integrated humoral and T cell responses in ovarian cancer," Proceedings of the National Academy of Sciences of the United States of America, vol. 104, no. 31, pp. 12837-12842, 2007.

[30] D. Atanackovic, N. K. Altorki, Y. Cao et al., "Booster vaccination of cancer patients with MAGE-A3 protein reveals long-term immunological memory or tolerance depending on priming," Proceedings of the National Academy of Sciences of the United States of America, vol. 105, no. 5, pp. 1650-1655, 2008.

[31] S. Cascinu, V. Georgoulias, D. Kerr, T. Maughan, R. Labianca, and M. Ychou, "Colorectal cancer in the adjuvant setting: perspectives on treatment and the role of prognostic factors," Annals of Oncology, vol. 14, supplement 2, pp. ii25-ii29, 2003.

[32] T. Watanabe, T.-T. Wu, P. J. Catalano et al., "Molecular predictors of survival after adjuvant chemotherapy for colon cancer," The New England Journal of Medicine, vol. 344, no. 16, pp. 1196-1206, 2001.

[33] K. W. Hunter, N. P. S. Crawford, and J. Alsarraj, "Mechanisms of metastasis," Breast Cancer Research, vol. 10, no. 1, article S2, 2008 . 
[34] U. Kong, J. Koo, K. Choi, J. Park, and H. Chang, “The expression of GAGE gene can predict aggressive biologic behavior of intestinal type of stomach cancer," Hepato-Gastroenterology, vol. 51, no. 59, pp. 1519-1523, 2004.

[35] T. Shiraishi, N. Terada, Y. Zeng et al., "Cancer/Testis antigens as potential predictors of biochemical recurrence of prostate cancer following radical prostatectomy," Journal of Translational Medicine, vol. 9, no. 1, article 153, 2011.

[36] Y. Zeng, Y. He, F. Yang et al., “The cancer/testis antigen prostateassociated gene 4 (PAGE4) is a highly intrinsically disordered protein," Journal of Biological Chemistry, vol. 286, no. 16, pp. 13985-13994, 2011.

[37] J. Tammela, A. A. Jungbluth, F. Qian et al., "SCP-1 cancer/testis antigen is a prognostic indicator and a candidate target for immunotherapy in epithelial ovarian cancer," Cancer Immunity, vol. 4, article 10, 2004. 


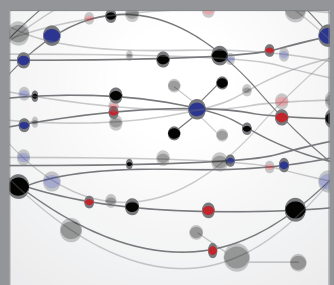

The Scientific World Journal
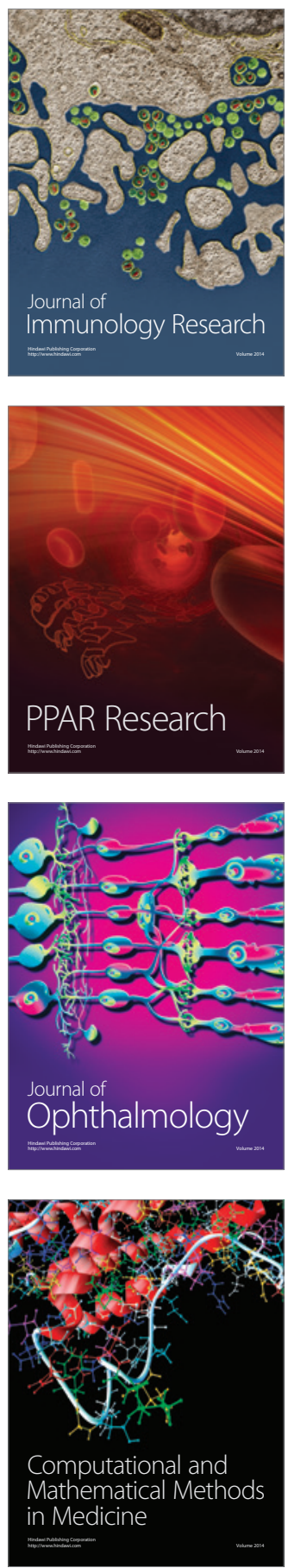

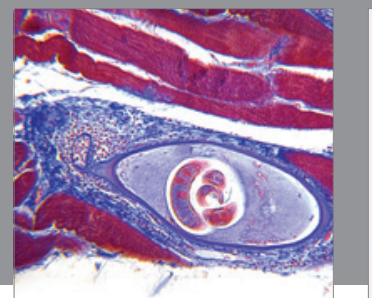

Gastroenterology

Research and Practice
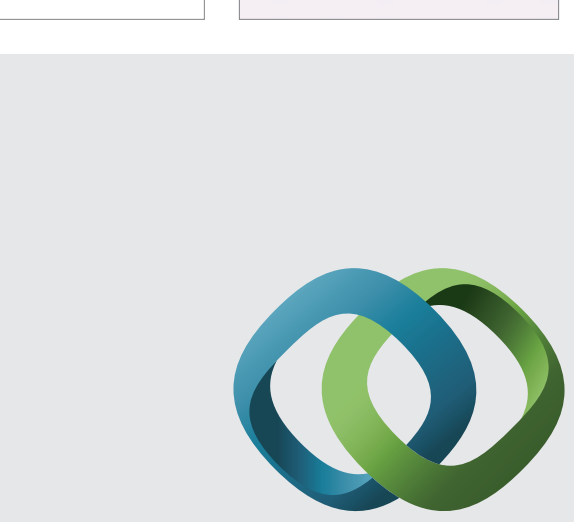

\section{Hindawi}

Submit your manuscripts at

http://www.hindawi.com
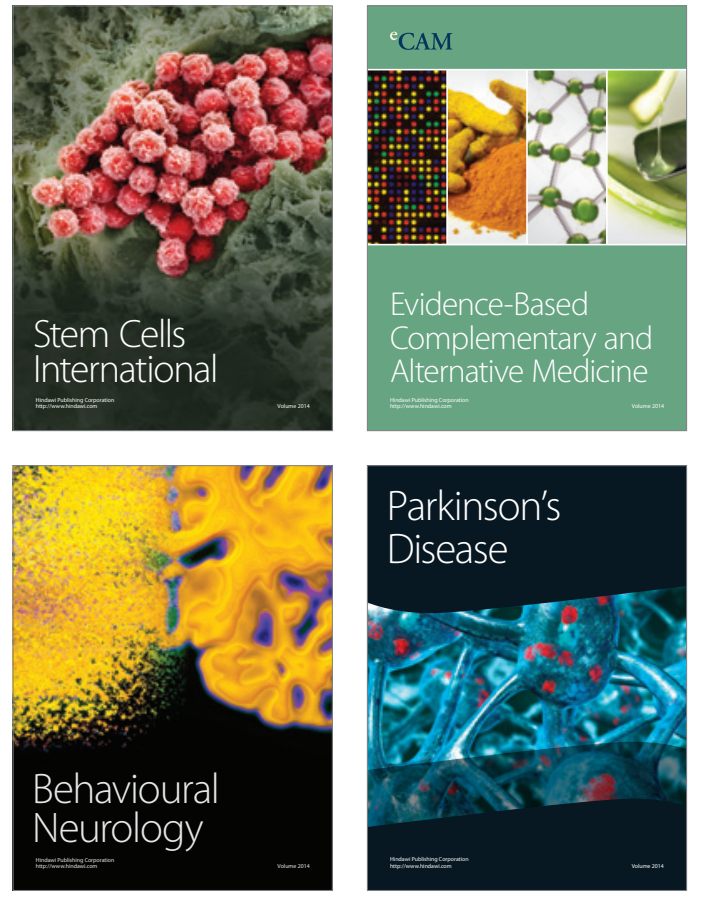
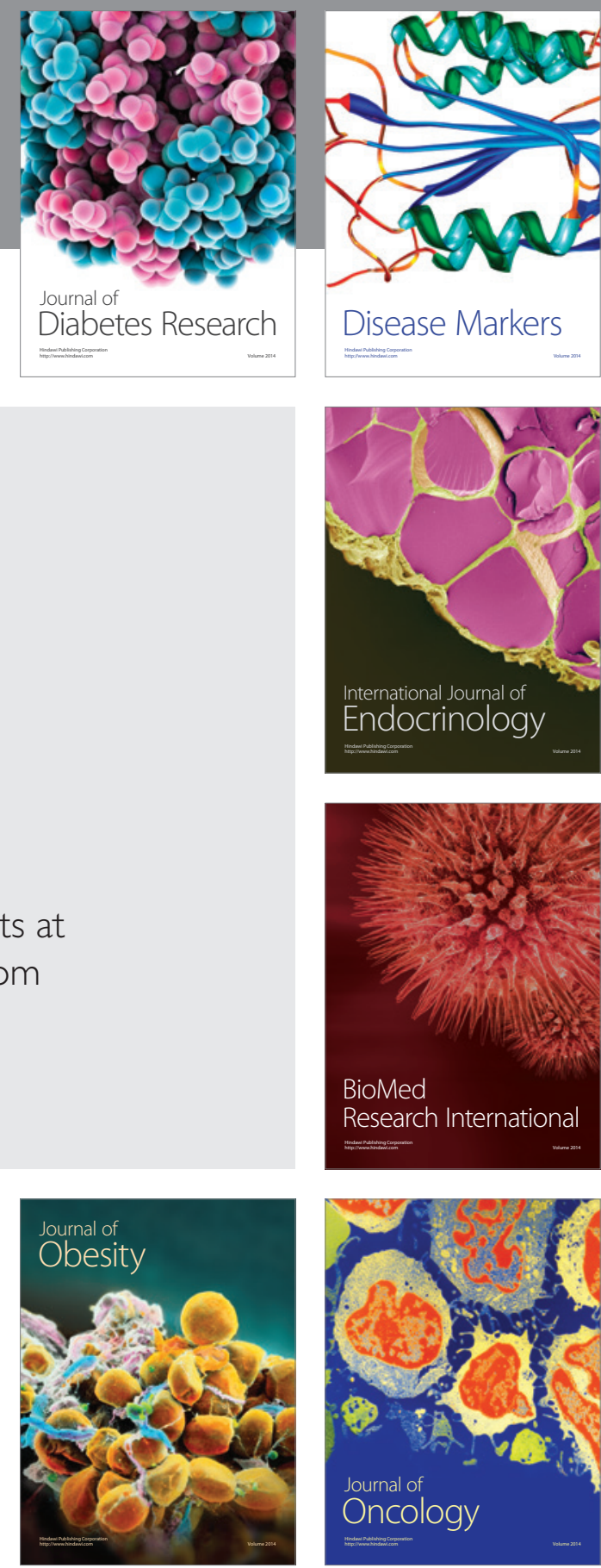

Disease Markers
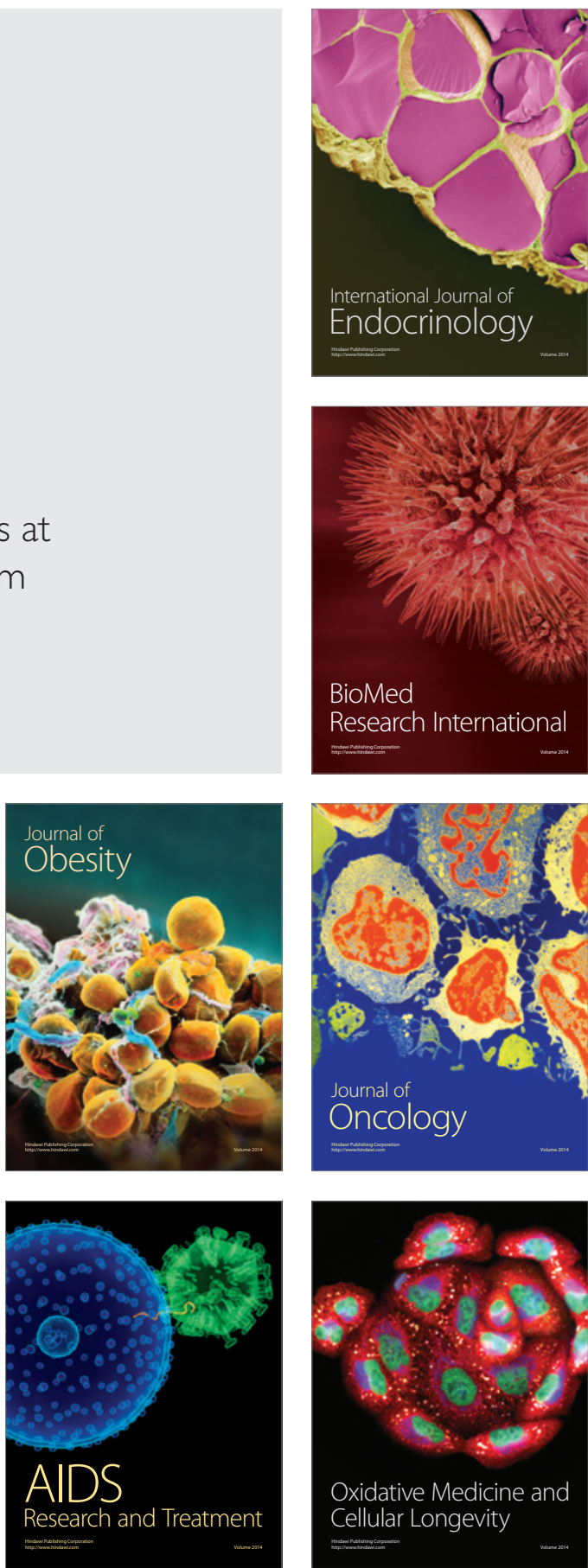